\title{
El profesor de Historia y el debate entre los saberes didácticos y disciplinares
}

\section{The history teacher and the debate between didactic and disciplinary knowledge}

\author{
Adolfo Alejandro Díaz Pérez \\ Doctorado en Educación e Intervención Social \\ Facultad de Educación e Idiomas \\ Universidad Nacional Autónoma de Nicaragua, \\ Managua (UNAN-Managua) \\ https://orcid.org/0000-0002-4295-4094 \\ adolfoalejandro73@gmail.com
}

\section{Referencia}

Díaz Pérez, A. A. (2021). El profesor de Historia y el debate entre los saberes didácticos y disciplinares. Revista Guatemalteca de Educación Superior, 4(1), pp 83-92.

DOI: https://doi.org/10.46954/revistages.v4i1.56

Recibido: 15/11/2020

Aceptado: 16/01/2021

\section{Resumen}

El dominio de los saberes didácticos y disciplinares es uno de los temas más discutidos en el aula de clase en los procesos de formación inicial del profesorado, en esta etapa, se confrontan dos tipos de concepciones: la primera tiene que ver con quienes sostienen que el saber pedagógico es suficiente para impartir cualquier disciplina escolar, y la segunda está asociada con aquellos que suponen que poseer vastos conocimientos de una ciencia es suficiente para impartir tal disciplina. Con base en esto, el presente ensayo tiene el objetivo de destacar la importancia de la complementariedad de los saberes didácticos y disciplinares en la enseñanza de la Historia en Educación Secundaria. Por consiguiente, en su contenido se abordan temáticas como el mito de "quien más sabe, más enseña", la transposición didáctica y la complementariedad de saberes. Los 
Palabras clave:

historia, saberes didácticos, saberes disciplinares, profesorado.

\section{Keywords:}

history, didactic knowledge, disciplinary knowledge, teaching resultados expuestos permiten afirmar que, para enseñar Historia y cualquier otra ciencia social, es indispensable el dominio de la ciencia, pero también hay que saberla enseñar para generar aprendizajes significativos en el estudiantado.

\section{Abstract}

The mastery of didactic and disciplinary knowledge is one of the most discussed topics in the classroom in the initial teacher training processes. At this stage, two types of conceptions are confronted: the first has to do with those who argue that pedagogical knowledge is sufficient to teach any school discipline, and the second is associated with those who assume that possessing vast knowledge of a science is sufficient to teach that discipline. Based on this, this essay aims to highlight the importance of the complementarity of didactic and disciplinary knowledge in the teaching of History in Secondary Education. Therefore, its content addresses issues such as the myth of "he who knows more, teaches more", didactic transposition and the complementarity of knowledge. The results presented allow us to affirm that, in order to teach History and any other social science, it is essential to master the science, but it is also necessary to know how to teach it in order to generate significant learning in the students.

\section{Introducción}

El debate entre los saberes didácticos y disciplinares es uno de los primeros tópicos que se suelen discutir en las aulas de clases de formación inicial del profesorado, esto debido a dos ideas previas que comúnmente el estudiantado trae consigo: la primera tiene que ver con aquellos que piensan que el saber pedagógico es suficiente para ser profesor e impartir clase, y la segunda razón está asociada con quienes suponen que poseer vastos conocimientos de una ciencia es suficiente para ser profesor e impartir tal disciplina. No obstante, ninguna afirmación es totalmente válida, sin embargo, esta discusión académica genera un espacio reflexivo que favorece la maduración inicial del pensamiento pedagógico. 
Lo cierto es que esta problemática no termina con una discusión académica en el aula de clase, sino que ambas ideas se convierten en concepciones que perduran durante largo tiempo, y al hacer referencia propiamente al profesor de Historia, esta problemática se manifiesta cuando directivos escolares piensan que esta asignatura puede ser impartida por cualquier docente que tenga conocimientos de pedagogía o por todo aquel que tenga amplios conocimientos históricos. Con base en estas concepciones paradójicas que existen sobre el quehacer de la docencia en el área de Historia, se ha escrito el presente ensayo con el objetivo de destacar la importancia que tiene la complementariedad de los saberes didácticos y disciplinares en la formación docente y en el proceso de enseñanza y aprendizaje.

\section{El mito de "quien más sabe, más enseña"}

En las realidades escolares existen diversas prácticas pedagógicas que invitan con urgencia a debatir acerca de los saberes didácticos y disciplinares en la formación docente, y una de las conjeturas más comunes sostenidas alrededor de la enseñanza de la Historia es creer que "el que sabe más de Historia es el más apropiado para impartir esta asignatura", lo cual deja a la deriva un conjunto de interrogantes que conllevan a reflexionar sobre lo siguiente: ¿Es necesario haber tenido una formación didáctica para impartir la asignatura de Historia? ¿El saber científico-disciplinar de la Historia es suficiente para llevar a cabo procesos de enseñanza y aprendizaje en un aula de clase? ¿Qué aportes brinda el saber didáctico a expertos en Historia que pretenden enseñar su disciplina a otros?

Estas preguntas parecieran haber sido respondidas acertadamente cuando en las escuelas de secundarias, particularmente, se ve impartiendo clases de Historia a profesores egresado de disciplinas como Matemáticas, Lengua y Literatura, Lengua Extranjera, Ciencias Naturales, o de las distintas especialidades de la Pedagogía, o bien, de otras ciencias sociales como la Antropología, Geografía, Economía o Filosofía; sin embargo, si esto fuese cierto, entonces no existiese tanta apatía del estudiantado hacia el aprendizaje de las ciencias 
sociales, y tampoco hubiesen engorrosas expresiones que ponen de manifiesto los problemas didácticos suscritos en esta área del conocimiento.

No obstante, es evidente que este tipo de concepciones academicistas forman parte de la cultura escolar $y$, sin duda alguna, están bien posicionadas entre los profesores. Sobre esto es oportuno detenerse a reflexionar en la afirmación de TovarGálvez y García Contreras (2012), cuando aseguran que existen "docentes que son profesionales y que conocen su disciplina, pero que no necesariamente aprendieron como enseñarla" (p.883), es decir, se reconoce que en el proceso de enseñanza y aprendizaje interfieren dos componentes esenciales que forman parte de una misma práctica pedagógica: El dominio del saber científico-disciplinar y del saber didáctico.

La falta de reconocimiento de esta complementariedad de saberes es uno de los problemas más complejos dentro del profesorado, y cuando no ha sido superado, esto logra repercutir en la metodología didáctica con la cual estos llevan a cabo los procesos de aprendizajes, por ejemplo, quien asume que el saber científico, o bien, el saber didáctico, es suficiente para impartir Historia, suele desarrollar un modelo didáctico academicista y aplicar metodologías narrativas y estrategias didácticas como conferencias magistrales, dictados y explicación de contenidos, y en la forma de evaluación resultan ser más severos, pues estos atiborran las evaluaciones escritas de preguntas conceptuales con la pretensión de que el estudiante reproduzca fielmente los contenidos de la asignatura.

Es decir, esto ejemplifica que los procesos de enseñanza y aprendizaje exigen más que el dominio de los contenidos de la asignatura que se imparte, por esto, el profesorado que únicamente se preocupa por dominar ampliamente los saberes científicos-disciplinares tendrá falencias metodológicas y didácticas al momento de ejercitar la enseñanza en el aula de clase, su práctica docente se caracterizará, como bien lo describen Tovar-Gálvez y García Contreras (2012), por la aplicación de "métodos educativos tradicionales en los que se privilegia la memorización de contenidos, el aprendizaje individual y la reproducción en la evaluación de los aprendizajes" (p.883). 
Estas concepciones academicistas del quehacer docente imprimen una visión pedagógica basada en paradigmas conductistas, modelos memoristas y enfoques didácticos bancarios que reducen el aprendizaje a la repetición de fechas, narración de sucesos y memorización de personajes, siendo el estudiante un almacén de información que no logra desarrollar sus capacidades analíticas, interpretativas y críticas; por otra parte, el profesorado se convierte en una especie de experto académico que llega al aula de clase a exponer sus amplios saberes históricos, pero sin lograr crear andamiajes didácticos que faciliten la construcción del conocimiento de manera significativa.

Como consecuencia de prescindir de las aportaciones de la didáctica de la historia, en el currículo escolar las asignaturas de ciencias sociales, particularmente la Historia, ostenta poco atractivo e importancia para el estudiantado, y como consecuencia se encuentra en desventaja con relación a aquellas disciplinas científicas que por sus fortalezas didácticas sí han logrado posicionar entre las más útiles, necesarias e importantes para los y las estudiantes. Sobre esto, bastaría con revisar los resultados del reciente estudio de Gómez y colaboradores (2016), cuyos resultados proporcionan hallazgos significativos acerca de la formación didáctica del profesorado y su incidencia en la percepción que los estudiantes tienen sobre la enseñanza de la Historia en la educación escolar.

Estas paradojas alrededor de las concepciones que se tienen sobre los saberes didácticos y disciplinares en la enseñanza de la Historia, también ha llevado a González Gallego (2010) a señalar enfáticamente que muchos profesores "creen que para enseñar una ciencia específica basta con conocerla y que de esa propia ciencia específica y de nuestro conocimiento sobre ella, nace todo lo necesario para poder enseñarla eficazmente" (p.4), pero como contra parte, también ha llevado a la comunidad de académicos educativos a realizar más aportes desde las didácticas específicas a fin de generar espacios de aprendizajes mediados con metodologías acordes a la naturaleza de cada disciplina científica. 
Por lo tanto, la hipótesis de "quien más sabe, más enseña" no tiene validez en los procesos de enseñanza y aprendizaje. En la asignatura Historia es insuficiente que el profesor exponga mediante extensas e interminables conferencias sus amplios conocimientos de acontecimientos y personajes históricos, por el contrario, se requiere de una oportuna mediación didáctica para que el profesorado convierta el saber científico en un saber posible de ser aprendido y aplicado a nuevas realidades, y esto solamente se logra haciendo uso de estrategias didácticas adecuadas, incorporando recursos y materiales didácticos en el aprendizaje, y efectuando una evaluación no reproductiva de saberes sino analítica, interpretativa y crítica; es decir, el saber didáctico es imprescindible para generar aprendizajes significativos en los procesos de enseñanza y aprendizaje.

\section{La transposición didáctica}

Lo que se ha descrito anteriormente tiende a apuntar directamente hacia el concepto de Transposición didáctica, el cual es uno de los términos que ineludiblemente debe formar parte del glosario pedagógico del profesorado activo y en formación, sin embargo, es poco conocido entre ellos; pero también, este término está muy relacionado con las llamadas didácticas específicas en donde los aportes provenientes de las diversas áreas de las ciencias de la educación han originado un crecimiento vertiginoso en el campo específico de la didáctica de las ciencias sociales y, en lo particular, en la didáctica de la historia.

Con base en esto, se define la transposición didáctica de acuerdo a los criterios de Valeta y colaboradores (2021, p.171), quienes la conciben como un "proceso de conciliaciones ejecutadas a los saberes para que el conocimiento ilustrado se convierta en conocimiento a enseñary finalmente se convierta en conocimiento enseñado", en otras palabras, los mismos autores insisten en que "la transposición didáctica transforma una disciplina en objeto de conocimiento, y por tanto, los diferentes saberes disciplinares se pueden transformar en saberes a ser enseñados y aprendidos", es decir, este concepto plantea consigo la acción de mediación didáctica que el profesorado debe de realizar sobre los saberes de la ciencia que enseña. 
En la formación inicial del profesorado, la transposición didáctica ha de ser uno de los primeros retos que se han de tomar para lograr la comprensión del quehacer docente desde la naturaleza de lo que se enseña. Este concepto permite que el estudiantado de las distintas disciplinas de la pedagogía cambie la naturaleza academicista de sus metodologías, en su mayoría narrativas y demostrativas, y construyan nuevas estructuras cognitivas que los conlleven a la puesta en marcha de procesos de enseñanza y aprendizaje mediados a través del uso de estrategias y recursos didácticos oportunos, y del desarrollo de procedimientos y acciones didácticas pertinentes a los contenidos y a la didáctica de la historia.

En vista de esto, esta discusión académica alrededor del concepto de transposición didáctica, abre enormes posibilidades para quienes ejercen docencia puedan realizar replanteamientos a aquellas prácticas pedagógicas academicista que aún se ciñen a anquilosadas concepciones que suponen que para enseñar una asignatura es suficiente tener amplios conocimientos de la misma, contrario a esto, el profesorado ha de plegarse a la idea de que no basta con saber mucho de historia y de otras ciencias sociales para saber enseñarla, sino que la preparación didáctica es fundamental para desarrollar procesos de enseñanza y aprendizajes eficaces en el aula de clase.

\section{La complementariedad de saberes}

La tendencia que se ha venido definiendo alrededor de los saberes didácticos y disciplinares en el quehacer del profesor de Historia, apunta hacia un modelo basado en la complementariedad de saberes en donde el dominio de sus conocimientos disciplinares, es decir, sus periodizaciones, acontecimientos, personajes, método de investigación y su interrelación con otras ciencias, se conjugan con el dominio didáctico de la misma, es decir, con su modelo didáctico (narrativo, explicativo, sociocrítico), las metodologías de enseñanza (planificación, estrategias, evaluación) y las finalidades de la enseñanza de la Historia. En palabras de Prats (2002), "la labor del profesorado (...) requiere, además del saber científico (...) el dominio de habilidades docentes específicas" (p.166). 
Y es aquí el punto de partida que debe hacer eco en los académicos que dirigen procesos de formación inicial del profesorado en escuelas especializadas, institutos técnicos y Centros de Educación Superior, pues a nivel curricular se requiere de un balance proporcional que a los futuros profesores les permita ser competentes tanto en lo disciplinar como en lo didáctico. En este sentido, la didáctica de las ciencias sociales desde estos centros de formación docente, juega un papel importante en la reconfiguración de la visión academicista que se ha venido construyendo sobre el profesor de Historia.

No obstante, queda explícitamente expresado que indistintamente del origen de la formación profesional que tenga el profesorado de Historia en los centros escolares de Educación Secundaria, es indispensable que este ostente dominio de los conocimientos histórico y dominio de los conocimientos didácticos de la historia, para así llevar a cabo procesos de enseñanza y aprendizaje significativos en el aula de clase. Esta visión plantea un reto importante para los académicos que se desempeñan en el área curricular y de formación docente, para el mismo profesorado en formación y para aquellos que ejercen la profesión, puesto que este debate invita a replantear la metodología didáctica de la Historia y a transitar de un modelo academicista a un modelo innovador, creativo, participativo y contextual.

\section{Conclusión}

La discusión dirigida en el transcurso de este ensayo señala enfáticamente que la formación disciplinar a espaldas de la didáctica es insuficiente para desarrollar procesos de enseñanza y aprendizajes exitosos en el aula de clase. Sin embargo, esta es una problemática presente en la formación inicial y continua del profesorado, y ha generado que en la enseñanza de la Historia se alojen metodologías narrativas y explicativas propias del modelo tradicional. No obstante, a nivel curricular esto sigue siendo un desafío emergente para los académicos que aún no lograr superar concepciones pedagógicas academicistas y enciclopedistas. 
La hondura de este debate entre los saberes didácticos y disciplinares del profesor de Historia, permiten reafirmar la hipótesis que Pagés (2001), uno de los pioneros en el área de la Didáctica de las Ciencias Sociales, claramente planteó: "Para enseñar historia y ciencias sociales se ha de saber historia y ciencias sociales, sin duda, pero también hay que saber enseñarlas" (p.285). Por consiguiente, la complementariedad entre ambos tipos de saberes son premisas esenciales para llevar a cabo procesos didácticos exitosos en la asignatura de Historia.

\section{Referencias}

Gómez, C., Rodríguez, R. y Mirete, A. (2016, septiembre). Percepción de la enseñanza de la historia y concepciones epistemológicas. Una investigación con futuros maestros. Revista Complutense de Educación, 29(1):237-250. https://doi.org/10.5209/RCED.52233

González Gallego, I. (2010). Prospectiva de las Didácticas Específicas, una rama de las Ciencias de la Educación para la eficacia en el aula. Perspectiva Educacional, 49(1):1-31. https://www.redalyc.org/pdf/3333/333327288001.pdf

Pagés, J. (2001). ¿Hacia dónde va la enseñanza de la historia y de las ciencias sociales? Apuntes para la comprensión de un debate. Éndoxa: series filosóficas, (14): 261-288. https://doi.org/10.5944/endoxa.14.2001.5027

Prats, J. (2002). La formación inicial del profesorado de Educación Secundaria: Reflexiones para un nuevo modelo. En Nuevos horizontes en la formación del profesorado de Ciencias Sociales (pp.163-178). Libros Activos, S.L. https://dialnet.unirioja.es/servlet/libro?codigo=5789

Tovar-Gálvez, J., y García Contreras, G. (2012, octubre). Investigación en la práctica docente universitaria: obstáculos epistemológicos y alternativas desde la Didáctica General Constructivista. Educação e Pesquisa, 38(4):881-895. https://doi.org/10.1590/S151797022012000400007 
Valeta, A., Torres, G., Mendoza, Y. y De la Hoz, S. (2021, enero). Transposición didáctica y la perspectiva crítica de Pablo Freire. Encuentros, (13):168-176. https://encuentros. unermb.web.ve/index.php/encuentros/article/ view/132/148

\section{Sobre el autor}

\section{Adolfo Alejandro Díaz Pérez}

Es profesor e investigador de la Facultad de Educación e Idiomas de la Universidad Nacional Autónoma de Nicaragua, Managua (UNAN-Managua). Es Licenciado en Ciencias de la Educación con mención en Ciencias Sociales y máster en Educación e Intervención Social, y autor de diversas publicaciones científicas en el área de la Didáctica de las Ciencias Sociales e Historia Oral.

Copyright (c) Adolfo Alejandro Díaz Pérez

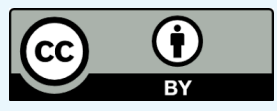

Este texto está protegido por una licencia CreativeCommons 4.0.

Usted es libre para compartir, copiar y redistribuir el material en cualquier medio o formato y adaptar el documento, remezclar, transformar y crear a partir del material para cualquier propósito, incluso comercialmente, siempre que cumpla la condición de atribución: usted debe reconocer el crédito de una obra de manera adecuada, proporcionar un enlace a la licencia, e indicar si se han realizado cambios. Puede hacerlo en cualquier forma razonable, pero no de forma tal que sugiera que tiene el apoyo del licenciante o lo recibe por el uso que hace. 